\title{
DIFFERENTIABILITY OF FRACTAL CURVES
}

\author{
CHRISTOPH BANDT AND ALEXEY KRAVCHENKO
}

\begin{abstract}
While self-similar sets have no tangents at any single point, self-affine curves can be smooth. We consider plane self-affine curves without double points and with two pieces. There is an open subset of parameter space for which the curve is differentiable at all points except for a countable set. For a parameter set of codimension one, the curve is continuously differentiable. However, there are no twice differentiable self-affine curves in the plane, except for parabolic arcs.
\end{abstract}

MSC classification: Primary 28A80, Secondary 26A27, 28A75

Christoph Bandt, Alexey Kravchenko

Institute for Mathematics and Informatics

Arndt University

17487 Greifswald, Germany

e-mail: bandt@uni-greifswald.de, alexey14@gmail.com

A.K. was supported by grant RFBR 09-01-90202-Mong_a and grant ADTP "Development of Scientific Potential of Higher Education" 2.1.1/3707 (Russia). Most work was done when A.K. was employed at Greifswald University. 


\section{Overview}

I. It is well-known that fractals are not differentiable. Nevertheless some fractals, like the Appolonian gasket, or parabolic Julia sets, possess tangents at some of their points. For self-similar sets

$$
A=f_{1}(A) \cup \ldots \cup f_{m}(A)
$$

where the $f_{i}$ are contracting similarity maps on $\mathbb{R}^{n}[6,5,3]$, there are no such exception points:

Theorem 1. (i) If $A$ is a self-similar set which spans $\mathbb{R}^{n}$ and $x \in A$, there does not exist a tangent hyperplane of $A$ at $x$.

(ii) If $\mu$ is a self-similar measure and $x \in \operatorname{supp} \mu$, there does not exist an approximate tangent hyperplane of $\mu$ at $x$ in the measure-theoretical sense.

Precise definitions and the proof are given in Section 2. Related results on selfconformal sets with separation condition can be found in Käenmäki [7, 8] and the references given there. Theorem 1 directly extends to self-conformal sets without separation condition - see Remark 6.

II. Self-affine curves, however, can be smooth, as shown in [10]. Here we prove that differentiability of self-affine curves is not an exception, but a rather generic phenomenon. We shall consider self-affine curves $J$ in the plane with two pieces:

$$
J=f_{1}(J) \cup f_{2}(J)
$$

where $f_{1}, f_{2}$ are contracting affine maps in $\mathbb{R}^{2}$ with positive eigenvalues and with fixed points $e_{1}, e_{2}$ respectively, and $f_{2}\left(e_{1}\right)=f_{1}\left(e_{2}\right)$. In Section 3 we show that the structure of $J$ is determined, up to an affine coordinate transformation, by the eigenvalues $\lambda_{1}, \nu_{1}$ of $f_{1}$ and $\lambda_{2}, \nu_{2}$ of $f_{2}$. We take $\lambda_{i} \geq \nu_{i}>0$, exclude similarity maps, and assume that the eigenvectors associated with $\lambda_{1}$ and $\lambda_{2}$ do not coincide. In Section 3, these conditions are stated more technically as (1) and (2).

Theorem 2. (i) Under the above assumptions, the curve $J$ is differentiable at all points $x \in J$ except for a countable set if

$$
\lambda_{1}+\nu_{2}<1 \quad \text { and } \quad \lambda_{2}+\nu_{1}<1 .
$$

(ii) If this condition holds, the curve is continuously differentiable if and only if the one-sided tangents at the intersection point $z=f_{2}\left(e_{1}\right)$ coincide:

$$
\nu_{1} \nu_{2}=\left(1-\lambda_{1}-\nu_{2}\right)\left(1-\lambda_{2}-\nu_{1}\right) \text {. }
$$

Figure 1 shows examples of everywhere and almost everywhere differentiable selfaffine curves. They were standardized to represent functions

$$
x_{2}=\psi\left(x_{1}\right) \quad \text { with } \quad \psi(-1)=\psi(1)=1 \quad \text { and } \quad \psi^{\prime}(-1)=-1, \psi^{\prime}(1)=1 .
$$

According to Theorem 2, there is a four-parameter family of such functions which are almost everywhere differentiable, and there is a three-parameter subfamily of continuously differentiable self-affine functions. In Section 3 we discuss the properties of such curves. In Section 4 we prove Theorem 2 . 

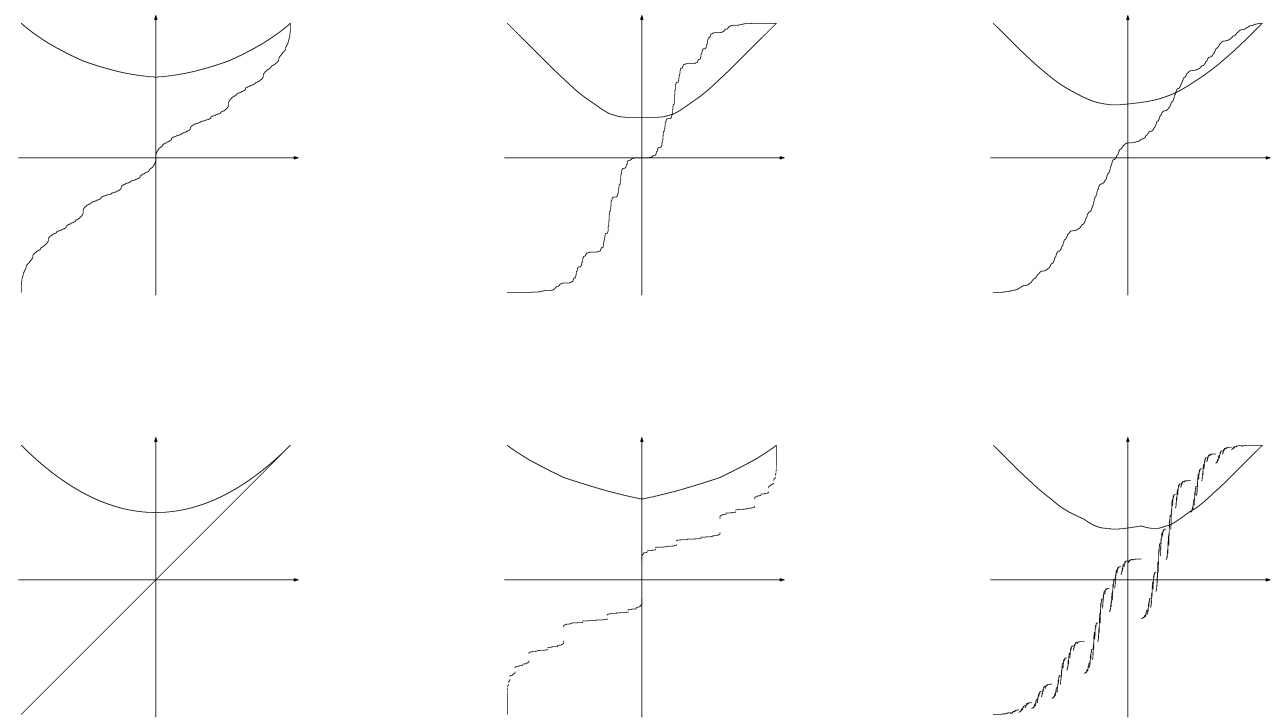

FiguRE 1. Upper row: several smooth self-affine functions with their derivatives. Lower row: parabola and two self-affine functions which are differentiable up to a countable set of points where only one-sided derivatives exist

III. Nevertheless, self-affine curves are not very smooth. With exception of segment and parabola, they are not $C^{2}$. The following theorem is proved in Section 5 .

Theorem 3. (i) If a plane curve $J$, parametrized as $x=\phi(t), \phi:[0,1] \rightarrow J$, is two times continuously differentiable with $\phi(0)=0$ and $\phi^{\prime \prime}(0) \neq 0$, and there exists a contractive linear map $h \neq 0$ which maps $J$ into itself, then $J$ is a parabolic arc.

(ii) Except for parabolic arcs and segments, there are no twice continuously differentiable self-affine curves in the plane.

\section{No TANGENTS TO SELF-SIMILAR SETS}

An affine subspace $V$ of $\mathbb{R}^{n}$ is tangent to a set $B$ at a point $x \in V \cap B$ if for each $\delta>0$ there is an $\epsilon>0$ such that

$$
\frac{\operatorname{dist}(b, V)}{|x-b|}<\delta \text { for all } b \in B \backslash\{x\} \text { with }|x-b|<\epsilon .
$$

Here dist $(b, V)=\inf \{|b-v| \mid v \in V\}$. In other words, all chords from $x$ to a point $b \in B \cap U_{\epsilon}(x)$ should subtend an angle smaller $\alpha=\arcsin \delta$ with $V$.

Lemma 4. Let $A$ be a bounded set which spans $\mathbb{R}^{n}$, and let $a_{0}, a_{1}, \ldots, a_{n}$ be $n+1$ points of $A$ in general position. Then there is an $\phi>0$ such that for all $y \in A$ and each hyperplane $V$ through $y$, there exists $i \in\{0,1, \ldots, n\}$ such that the chord ya forms an angle $\geq \phi$ with $V$. 
Proof. Let $B$ be a closed ball with radius $R$ which lies within the simplex $S=$ conv $\left\{a_{0}, \ldots, a_{n}\right\}$. Now take an arbitrary point $y \in A$ and an arbitrary hyperplane $V$ through $y$. Let $W=\left\{x \in \mathbb{R}^{n} \mid\right.$ dist $\left.(x, V)<R\right\}$. Then $B$ is not a subset of $W$. Since $W$ is convex, and $B$ lies in the convex hull of the $a_{j}$, it follows that there is at least one point $a_{i}$ outside $W$. For the angle $\beta$ between $y a_{i}$ and $V$ we have

$$
\sin \beta=\frac{\operatorname{dist}\left(a_{i}, V\right)}{\left|a_{i}-y\right|} \geq \frac{R}{\operatorname{diam} A} .
$$

We proved the lemma for $\phi=\arcsin \frac{R}{\operatorname{diam} A}$.

Proof of Theorem 1 (i). The lemma prevents approximation by a plane on global scale. We show that for a self-similar set $A$, Lemma 4 remains true in small neighborhoods. Let $\delta=\sin \frac{\phi}{2}$, and let $x \in A$ and $\epsilon>0$ be arbitrarily chosen. There is a small piece $A_{\mathbf{j}}=f_{\mathbf{j}}(A)$ of $A$ within $U_{\epsilon}(x)$. Here $\mathbf{j}=j_{1} \ldots j_{k}$ and $f_{\mathbf{j}}=f_{j_{1}} \ldots f_{j_{k}}$. The similarity map $f_{\mathbf{j}}^{-1}$ maps $A_{\mathbf{j}}$ onto $A$, and any hyperplane $V^{\prime}$ through $x$ onto a corresponding hyperplane $V$ through $y=f_{\mathbf{j}}^{-1}(x)$. Lemma 4 says that there is a chord $y a_{i}$ which subtends an angle $\geq \phi$ with $V$. Since the chord $x f_{\mathbf{j}}\left(a_{i}\right)$ within $U_{\epsilon}(x)$ subtends the same angle with $V^{\prime}$, the definition of tangent is not fulfilled for $V^{\prime}$ and $x$. So there is no tangent hyperplane to $A$ in $x$.

To deal with tangents of measures, we have to specify concepts. For a finite Borel measure $\mu$, the support supp $\mu$ contains all those points $y$ for which $\mu\left(U_{\epsilon}(y)\right)>0$ for all $\epsilon>0$. An affine subspace $V$ of $\mathbb{R}^{n}$ is an approximate tangent to $\mu$ at a point $x \in V \cap \operatorname{supp} \mu$ if for each $\delta>0$ there is an $\epsilon>0$ with

$$
\mu\left\{b \in U_{\epsilon}(x) \mid \frac{\operatorname{dist}(b, V)}{|x-b|}>\delta\right\} / \mu\left(U_{\epsilon}(x)\right)<\delta .
$$

Now we allow for chords $x b$ which subtend an angle $>\alpha=\arctan \delta$ with $V$. But the percentage of endpoints $b \in U_{\epsilon}(x)$ with this property, measured by $\mu$, should converge to zero with $\epsilon$. See Mattila [11], Chapter 15, for related concepts.

We are going to derive Theorem 1 (ii) from the above proof of (i). First we reformulate a global fact for arbitrary measures, giving a lower bound $\eta$ for the number of exceptions.

Lemma 5. Let $\mu$ be a probability measure on $\mathbb{R}^{n}$ such that $A=\operatorname{supp} \mu$ is bounded and contains $n+1$ points $a_{0}, \ldots, a_{n}$ in general position. Then there are $\psi>0, \gamma>$ $0, \eta>0$ such that for all $y \in \operatorname{supp} \mu$ and each hyperplane $V$ through $y$, there exists $i \in\{0,1, \ldots, n\}$ such that $W=U_{\gamma}\left(a_{i}\right)$ fulfils $\mu(W) \geq \eta$, and all chords $y w$ with $w \in W$ form an angle $\geq \psi$ with $V$.

Proof. In proving Lemma 4, we found $R, \phi$ such that for all $y, V$ there is $i$ such that dist $\left(a_{i}, V\right) \geq R$, and $y a_{i}$ and $V$ subtend an angle $\geq \phi$. Since $\tan \frac{\phi}{2}<\frac{1}{2} \tan \phi$, we can take $\gamma=\frac{R}{2} \sin \phi$ in order to ensure that every chord $y w$ with $w \in U_{\gamma}\left(a_{i}\right)$ subtends an angle $\geq \frac{\phi}{2}$ with $V$. With $\psi=\frac{\phi}{2}$ and $\eta=\min \left\{\mu\left(U_{\gamma}\left(a_{i}\right) \mid i=0, \ldots, n\right\}\right.$, the lemma holds true. 
A self-similar measure $\mu$ with probability vector $\left(p_{1}, \ldots, p_{m}\right)$ with $p_{i}>0, \sum p_{i}=1$ and contracting similarity maps $f_{i}$ is given by the equation

$$
\mu(B)=\sum_{i=1}^{m} p_{i} \mu\left(f_{i}^{-1}(B)\right) \quad \text { for Borel sets } B \subset \mathbb{R}^{n} .
$$

We require $\mu\left(\mathbb{R}^{n}\right)=1$. Then for given $p_{i}, f_{i}$ there is a unique $\mu$, and $\operatorname{supp} \mu$ is the self-similar set $A$ associated with the $f_{i}$ [5, 3].

Let $I=\{1, \ldots, m\}$, and let $I^{*}=\bigcup_{k=1}^{\infty} I^{k}$ denote the set of words $\mathbf{j}=j_{1} \ldots j_{k}$ on $I$, and $I^{\infty}$ the set of sequences $j_{1} j_{2} \ldots$ We write $f_{\mathbf{j}}=f_{j_{1}} \ldots f_{j_{k}}$ and $p_{\mathbf{j}}=p_{j_{1}}$. $\ldots \cdot p_{j_{k}}$. The product measure $\nu=\left(p_{1}, \ldots, p_{m}\right)^{\infty}$ on $I^{\infty}$ assigns to each cylinder set $C_{\mathbf{j}}=\left\{i_{1} i_{2} \ldots \mid i_{1} \ldots i_{k}=\mathbf{j}\right\}$ the value $\nu\left(C_{\mathbf{j}}\right)=p_{\mathbf{j}}$. There is the continuous address map $\pi: I^{\infty} \rightarrow A$ with $\pi\left(j_{1} j_{2} \ldots\right)=\bigcap_{k} A_{j_{1} \ldots j_{k}}$. The measure $\mu$ is the image measure of the product measure, $\mu=\nu \pi^{-1}$ [3].

Proof of Theorem 1 (ii). It remains to show the assertion of the lemma for arbitrary small neighborhoods $U_{\epsilon}(x)$ by using self-similarity. We consider the set $\mathcal{J}$ of all words $\mathbf{j}=j_{1} \ldots j_{k} \in I^{*}$ for which $A_{\mathbf{j}} \subset U_{\epsilon}(x)$ but $A_{j_{1} \ldots j_{k-1}} \not \subset U_{\epsilon}(x)$. Then

$$
\mu\left(U_{\epsilon}(x)\right)=\sum_{\mathbf{j} \in \mathcal{J}} p_{\mathbf{j}}
$$

because $\pi^{-1}\left(U_{\epsilon}(x)\right)$ is an open subset of $I^{\infty}$, that is, a countable union of disjoint cylinder sets, which are the $C_{\mathbf{j}}, \mathbf{j} \in \mathcal{J}$.

Now let a hyperplane $V^{\prime}$ through $x$ be given. For all $\mathbf{j} \in \mathcal{J}$ we apply $f_{\mathbf{j}}^{-1}$ with $y_{\mathbf{j}}=f_{\mathbf{j}}^{-1}(x)$ as in the proof of Theorem 1 (i). Lemma 5 yields a set $W_{\mathbf{j}} \subset A$ with $\mu\left(W_{\mathbf{j}}\right) \geq \eta$ such that all chords $y_{\mathbf{j}} w$ with $w \in W_{\mathbf{j}}$ subtend an angle $\geq \psi$ with $V_{\mathbf{j}}=f_{\mathbf{j}}^{-1}\left(V^{\prime}\right)$. Now the set

$$
W^{\prime}=\bigcup_{\mathbf{j} \in \mathcal{J}} f_{\mathbf{j}}\left(W_{\mathbf{j}}\right) \quad \text { fulfils } \quad \mu\left(W^{\prime}\right) \geq \sum_{\mathbf{j} \in \mathcal{J}} \eta p_{\mathbf{j}}=\eta \mu\left(U_{\epsilon}(x)\right) .
$$

Note that the measures of $f_{\mathbf{j}}\left(W_{\mathbf{j}}\right)$ have to be added even if the $W_{\mathbf{j}}$ overlap. Moreover, all chords $x w^{\prime}$ with $w^{\prime} \in W^{\prime}$ subtend an angle $\geq \psi$ with $V^{\prime}$. This holds for fixed $x, V^{\prime}$ with arbitrary $\epsilon$. Taking $\delta<\eta$, the condition for approximate tangent cannot be fulfilled.

Remark 6. It is not necessary that the mappings $f_{\mathbf{j}}$ between $A$ and the small pieces are similitudes. It is enough to require that there exists a constant $C$ such that for all $\mathbf{j} \in I^{*}$ and all angles $\beta=\angle a b c$ between points in $A$, the image angle fulfils $f_{\mathbf{j}}(\beta)=$ $\angle f_{\mathbf{j}}(a) f_{\mathbf{j}}(b) f_{\mathbf{j}}(c) \geq C \beta$. Thus Theorem 1 immediately extends to self-conformal sets as studied in [7, 8].

\section{Self-AfFine CURVES With two Pieces}

We shall consider two contracting affine mappings $f_{i}(x)=M_{i} x+v_{i}$ which are not similarity maps. So the eigenvalues $\nu_{i}, \lambda_{i}$ of $f_{i}$ must be real, and we further assume that they are positive: $\quad 0<\nu_{i} \leq \lambda_{i}<1 \quad$ for $i=1,2$.

The fixed points of the $f_{i}$ will now be taken as unit points $e_{1}=\left(\begin{array}{l}1 \\ 0\end{array}\right)$ and $e_{2}=\left(\begin{array}{l}0 \\ 1\end{array}\right)$ 

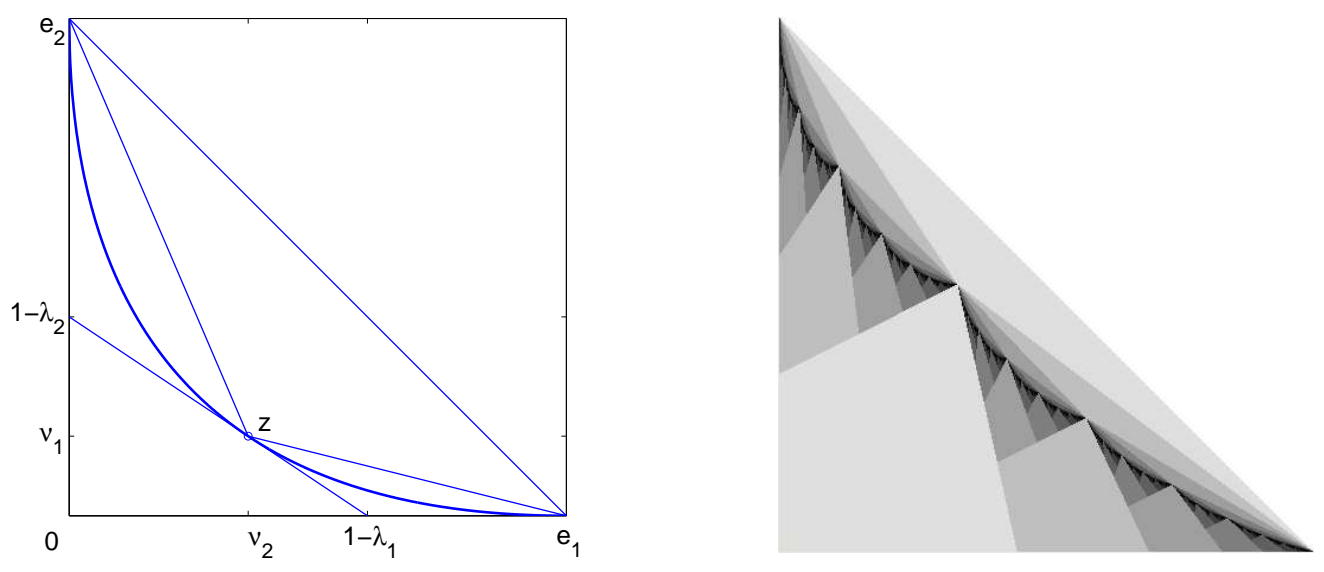

Figure 2. A smooth curve $(\alpha, \beta<0)$ and a non-differentiable curve $(\alpha<0, \beta>0)$ in the new coordinate system. The triangle $T=$ $\triangle 0 e_{1} e_{2}$ and its images under the $f_{i}$ are indicated.

of our coordinate system. The eigendirections of $M_{i}$ with respect to $\lambda_{i}$ are taken as axes. We assume that the eigendirections of $\lambda_{1}$ and $\lambda_{2}$ are not parallel, so that the axes do intersect.

Compared to the coordinate system of Figure 1, we turned our axes by 45 degrees. With these coordinates, our mappings have the form

$$
f_{1}(x)=\left(\begin{array}{cc}
\lambda_{1} & \alpha \\
0 & \nu_{1}
\end{array}\right) \cdot x+\left(\begin{array}{c}
1-\lambda_{1} \\
0
\end{array}\right), \quad f_{2}(x)=\left(\begin{array}{cc}
\nu_{2} & 0 \\
\beta & \lambda_{2}
\end{array}\right) \cdot x+\left(\begin{array}{c}
0 \\
1-\lambda_{2}
\end{array}\right) .
$$

The condition $f_{1}\left(\begin{array}{l}0 \\ 1\end{array}\right)=f_{2}\left(\begin{array}{l}1 \\ 0\end{array}\right)$ implies $\alpha=\nu_{2}+\lambda_{1}-1$ and $\beta=\nu_{1}+\lambda_{2}-1$, so that the vector of eigenvalues $\left(\lambda_{1}, \nu_{1}, \lambda_{2}, \nu_{2}\right)$ parametrizes all our possible self-affine curves. Moreover, the coordinates of the point $z=f_{1}\left(e_{2}\right)=f_{2}\left(e_{1}\right)$ are $\left(\begin{array}{l}\nu_{2} \\ \nu_{1}\end{array}\right)$.

If $\nu_{1}+\nu_{2}=1$, then $J$ will be the segment with endpoints $e_{1}, e_{2}$. In the case $\nu_{1}+\nu_{2}>1$ the set $J$ will not be a simple curve. Among others, $z$ will be a multiple point of the curve. Thus we shall require $\nu_{1}+\nu_{2}<1$, which means that $z$ lies in the interior of the triangle $T$ with vertices $0, e_{1}$, and $e_{2}$. Note that $\lambda_{1}+\nu_{2}<1$ in Theorem 2 implies $\nu_{1}+\nu_{2}<1$. Let us also note that in the special case $\nu_{1}=\lambda_{1}$ we get $\alpha<0$. Similarly, $\nu_{2}=\lambda_{2}$ implies $\beta<0$ so that we have no similarity maps.

Assumptions. The conditions of Theorem 2 are given in (1) above and (2) below. They are taken as assumptions for the rest of the paper.

$$
0<\nu_{i} \leq \lambda_{i}<1 \text { for } i=1,2, \quad \nu_{1}+\nu_{2}<1, \quad \alpha=\nu_{2}+\lambda_{1}-1, \quad \beta=\nu_{1}+\lambda_{2}-1
$$

The maps $f_{1}, f_{2}$ are said to fulfil the open set condition if there is an open set $U$ such that $f_{1}(U)$ and $f_{2}(U)$ are disjoint subsets of $U$ [12, 5, 3]. For self-similar curves, this condition is hard to verify even if we assume that $f_{1}(J) \cap f_{2}(J)$ consists of a single point only [1, 2]. In our setting, we get this condition for free. 
Proposition 7. The intersection of $f_{1}(J)$ and $f_{2}(J)$ consists of a single point. If $U$ denotes the interior of the triangle $T$ with vertices $\left(\begin{array}{l}0 \\ 0\end{array}\right),\left(\begin{array}{l}1 \\ 0\end{array}\right),\left(\begin{array}{l}0 \\ 1\end{array}\right)$ then $f_{1}, f_{2}$ fulfil the open set condition with $U$.

Proof. We just note that $f_{1}(T)$ is the triangle with vertices $e_{1}, z,\left(\begin{array}{c}1-\lambda_{1} \\ 0\end{array}\right)$, and $f_{2}(T)$ is the triangle with vertices $e_{2}, z,\left(\begin{array}{c}0 \\ 1-\lambda_{2}\end{array}\right)$. See Figure 2 .

Proposition 8. J is a simple curve without double points. If for the moment we write $f_{0}$ instead of $f_{2}$, an explicit homeomorphism $\varphi:[0,1] \rightarrow J$ is given by binary numbers: $\varphi(t)=\bigcap_{k=1}^{\infty} f_{i_{1} \ldots i_{k}}(J)$ for $t=\sum_{k=1}^{\infty} i_{k} \cdot 2^{-k}, i_{1}, i_{2}, \ldots \in\{0,1\}$.

Proof. This directly follows from Proposition 7. See [6], 3.5, compare also [1, 2], or [3], Chapter VIII.2. The point is that the two addresses $01111 \ldots=0 \overline{1}$ and $1 \overline{0}$ of the intersection point $z=J_{1} \cap J_{2}$ are the same as for the point $\frac{1}{2}$ when $[0,1]$ is considered as self-similar set with respect to $g_{0}(t)=\frac{t}{2}$ and $g_{1}(t)=\frac{t+1}{2}$.

Proposition 9. If $\lambda_{1}+\nu_{2} \leq 1$ and $\lambda_{2}+\nu_{1} \leq 1$ then $J$ is the graph of a strictly decreasing function $x_{2}=\psi\left(x_{1}\right)$ in our coordinate system. Thus $J$ is rectifiable, with one-dimensional Hausdorff measure $0<\mathcal{H}^{1}(J) \leq$ 2. The function $\psi$ is differentiable at almost all points $x_{1}$.

Proof. Let $W=\left\{\left(\begin{array}{c}w_{1} \\ w_{2}\end{array}\right) \mid w_{1} w_{2} \leq 0\right\}$ denote the cone consisting of the second and fourth quadrant of our coordinate system. Our assumptions say that $\alpha, \beta \leq 0$, so $W$ is invariant under the linear mappings $h_{i}(x)=M_{i} x$ corresponding to the $f_{i}$ in (1): $M_{i} w \in W$ for $w \in W$.

The curve $J$ is the graph of a decreasing function if $y-y^{\prime} \in W$ for all $y, y^{\prime} \in J$. (Then $y-y^{\prime}$ cannot be a multiple of $e_{2}$ (or $e_{1}$, respectively) because in such case all points between $y$ and $y^{\prime}$ must lie on that vertical segment which contradicts selfaffinity. See also Section 4.) Since $W$ is closed, and any $y \in J$ can be approximated by points of the form $f_{\mathbf{j}}\left(e_{i}\right)$ with $i \in I$ and $\mathbf{j} \in I^{*}$, it suffices to show the relation only for points $y, y^{\prime}$ of this form.

Clearly $e_{2}-e_{1}$ is in $W$. For $x-x^{\prime} \in W$ and $i=1,2$ we have $f_{i}(x)-f_{i}\left(x^{\prime}\right)=$ $M_{i}\left(x-x^{\prime}\right) \in W$. This implies $v=f_{\mathbf{j}}\left(e_{2}\right)-f_{\mathbf{j}}\left(e_{1}\right) \in W$ for every $\mathbf{j} \in I^{*}$. For arbitrary $k \in \mathbb{N}$ and $\mathbf{i}, \mathbf{j} \in I^{k}$, the difference $f_{\mathbf{j}}\left(e_{2}\right)-f_{\mathbf{i}}\left(e_{1}\right)$ is a finite sum of vectors of the form $v$, since in the $k$-th level of construction of $J$, the triangles $f_{\mathbf{j}}(T)$ and $f_{\mathbf{i}}(T)$ are connected by a finite chain of such triangles. Since finite sums of vectors in $W$ belong to $W$, we proved $f_{\mathbf{j}}\left(e_{2}\right)-f_{\mathbf{i}}\left(e_{1}\right) \in W$ for $\mathbf{i}, \mathbf{j} \in I^{k}$ and $y-y^{\prime} \in W$ for $y, y^{\prime} \in J$.

Since the curve $J$ is the graph of a function, its length is

$$
\mathcal{H}^{1}(J)=\sup \left\{\sum_{i=1}^{N}\left|y^{(i)}-y^{(i-1)}\right| \mid N \in \mathbb{N}, 0=y_{1}^{(0)}<y_{1}^{(1)}<\ldots<y_{1}^{(N)}=1\right\} .
$$

Since $\left|y^{(i)}-y^{(i-1)}\right| \leq\left|y_{1}^{(i)}-y_{1}^{(i-1)}\right|+\left|y_{2}^{(i)}-y_{2}^{(i-1)}\right|$ and $\sum_{l=1}^{N}\left|y_{1}^{(i)}-y_{1}^{(i-1)}\right|=\sum_{l=1}^{N} \mid y_{2}^{(i)}-$ $y_{2}^{(i-1)} \mid=1$, we get $\mathcal{H}^{1}(J) \leq 2$. The inequality $\mathcal{H}^{1}(J)>0$ holds since $J$ is connected, see [4, Lemma 3.2]. It is well-known that curves of finite length are differentiable 

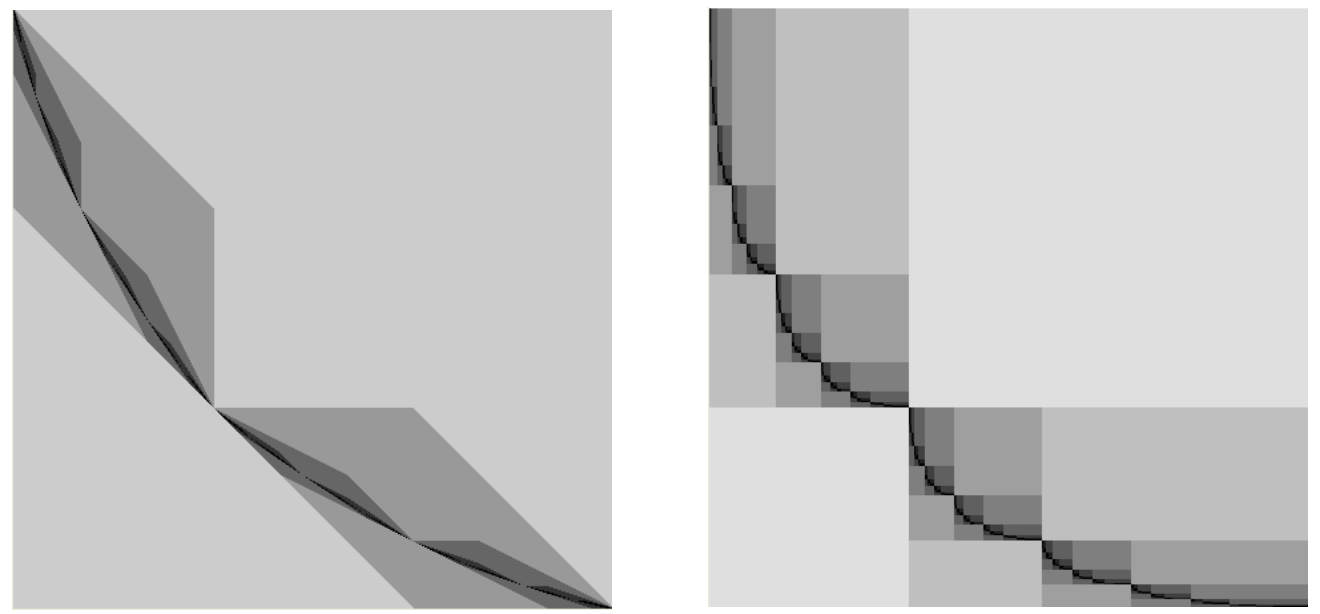

Figure 3 . The images of the unit square under the $f_{\mathbf{i}}$. The curve on the left is smooth, $\lambda_{i}=\nu_{i}=\frac{1}{3}$. Example 10 on the right has uncountable dense sets of points where the curve is differentiable and non-differentiable, $\lambda_{i}=\frac{2}{3}, \nu_{i}=\frac{1}{3}$.

$\mathcal{H}^{1}$-almost everywhere [4, Theorem 3.8], and monotone functions are differentiable at almost every real argument.

Example 10. The case $\lambda_{1}=\lambda_{2}=\frac{2}{3}, \nu_{1}=\nu_{2}=\frac{1}{3}, \alpha=\beta=0$, illustrated in Figure 1b, was studied by Käenmäki and Vilppolainen [9, Example 6.2]. J fulfils the conditions of Proposition 9 and thus has a tangent $\mathcal{H}^{1}$-almost everywhere although at all points $y=f_{\mathbf{j}}(z)$ the left-sided derivative is 0 and the right-sided one is $\infty$. We have $\mathcal{H}^{1}(J)=2$.

Let $\mu$ be the self-similar measure on $J$ induced by the product measure $\left(\frac{1}{2}, \frac{1}{2}\right)^{\infty}$ (see Section 2). Then at $\mu$-almost all points, $J$ does not possess a tangent.

Proof. We consider the rectangles $R_{\mathbf{j}}=f_{\mathbf{j}}\left([0,1]^{2}\right)$ for $\mathbf{j} \in I^{n}$, with side length $s_{\mathbf{j}}$ in direction $e_{1}$ and $t_{\mathbf{j}}$ in direction $e_{2}$. The ratio $r_{\mathbf{j}}=s_{\mathbf{j}} / t_{\mathbf{j}}$ of the side lengths is $2^{n_{\mathbf{j}}}$ where $n_{\mathbf{j}}=\#\left\{k \leq n \mid j_{k}=1\right\}-\#\left\{k \leq n \mid j_{k}=2\right\}$. Thus $-n \leq n_{\mathbf{j}} \leq n$. Probabilistic arguments will now show that for large $n$, most of the rectangles are very lengthy and slim.

When all words $\mathbf{j} \in I^{n}$ are assigned equal probability $2^{-n}$, then $n_{\mathbf{j}}$ is distributed like the endpoints of a symmetric random walk on the integers, with start in zero, after $n$ steps.

The two rectangles at $f_{i_{1} \ldots i_{k}}(z)$ correspond to the words $\mathbf{j}=i_{1} \ldots i_{k} 122 \ldots 2$ and $\mathbf{j}^{\prime}=i_{1} \ldots i_{k} 211 \ldots 1$. Thus $n_{\mathbf{j}} \rightarrow-\infty$ and $n_{\mathbf{j}^{\prime}} \rightarrow \infty$ for $n \rightarrow \infty$, which shows the assertion on one-sided derivatives.

Now we shall show that for large $n$, the sum of $\operatorname{diam} R_{\mathbf{j}}$ over $\mathbf{j} \in I^{n}$ is almost 2 , which by (3) implies $\mathcal{H}^{1}(J) \geq 2$. We let $n$ be even. Then $\mathbf{P}\left(n_{\mathbf{j}}=0\right) \approx \frac{1}{\sqrt{\pi n}}$ is the 
middle term of the binomial distribution for $n$ and $p=\frac{1}{2}$, so $\mathbf{P}\left(n_{\mathbf{j}} \leq 0\right)<\frac{1}{2}+\frac{1}{\sqrt{n}}$ holds for large $n$ (in fact for all even $n$ ).

For $n_{\mathbf{j}} \leq 0$ the square has the biggest horizontal side: $s_{\mathbf{j}}<\left(\frac{2}{9}\right)^{n / 2}$. Thus

$$
\sum_{n_{\mathbf{j}} \leq 0} s_{\mathbf{j}} \leq 2^{n}\left(\frac{1}{2}+\frac{1}{\sqrt{n}}\right)\left(\frac{2}{9}\right)^{n / 2} \leq\left(\frac{8}{9}\right)^{n / 2} \rightarrow 0 \quad \text { for } n \rightarrow \infty .
$$

This implies that $\sum_{n_{\mathbf{j}} \geq 1}$ diam $R_{\mathbf{j}} \geq \sum_{n_{\mathbf{j}} \geq 1} s_{\mathbf{j}} \rightarrow 1$. A similar calculation for the $t_{\mathbf{j}}$ yields $\sum_{n_{\mathbf{j}} \leq-1} \operatorname{diam} R_{\mathbf{j}} \rightarrow 1$ for $n \rightarrow \infty$. So we obtain $\mathcal{H}^{1}(J) \geq 2$.

For the last statement, we use the recurrence of the one-dimensional symmetric random walk. For almost all $j_{1} j_{2} \ldots \in I^{\infty}$, with respect to the product measure $\left(\frac{1}{2}, \frac{1}{2}\right)^{\infty}$, we have $n_{j_{1} \ldots j_{n}}=0$ for infinitely many $n$. That is, $\mu$-almost all points $y \in J$ lie in arbitrarily small squares $R_{\mathbf{j}}$. These points $y$ have arbitrary small neighborhoods which are geometrically similar to $J$. Using the argument of the proof of Theorem 1 in Section 2, we can conclude that $J$ does not admit a tangent at such points $y$.

In this example, the points of differentiability form an uncountable dense set with an uncountable dense complement. It turns out, however, that all tangent lines have only two directions, horizontal and vertical (cf. Section 4). In [9] the example was used to show that there are exactly two equilibrium measures. These measures are now obtained as image measures of the Lebesgue measure on the axes under the natural one-to-one map. They assign $s_{\mathbf{j}}$ and $t_{\mathbf{j}}$, respectively, to $R_{\mathbf{j}}$. The Hausdorff measure is just the sum of these two measures.

\section{Smooth SELF-AFFine CURVES}

Proposition 11. The curve $J$ has the two coordinate axes as tangents at $e_{1}$ and $e_{2}$, respectively. Thus $J$ has one-sided tangents at all points where two little pieces meet.

Proof. As neighborhoods of $e_{1}$ in $J$ we consider the sets $f_{1}^{k}(J)$ for large $k$. Since $f_{1}^{k}(J) \subset f_{1}^{k}(T)$, we just have to consider the angle $\beta_{k}$ of the triangle $f_{1}^{k}(T)$ at the vertex $e_{1}$ (cf. Section 1). From (1) we have

$$
f_{1}^{k}(x)=\left(\begin{array}{cc}
\lambda_{1}^{k} & \alpha_{k} \\
0 & \nu_{1}^{k}
\end{array}\right) \cdot x+\left(\begin{array}{c}
1-\lambda_{1}^{k} \\
0
\end{array}\right) .
$$

In the case $\lambda_{1}=\nu_{1}$, induction shows $\alpha_{k}=k \alpha \lambda_{1}^{k-1}$ where $\alpha_{1}=\alpha=\nu_{2}+\nu_{1}-1<0$. For $\lambda_{1}>\nu_{1}$, induction shows

$$
\alpha_{k}=\alpha \cdot \frac{\lambda_{1}^{k}-\nu_{1}^{k}}{\lambda_{1}-\nu_{1}}
$$

The relation $\alpha=\nu_{2}+\lambda_{1}-1<\lambda_{1}-\nu_{1}$ can be rewritten as $\alpha=\gamma\left(\lambda_{1}-\nu_{1}\right)$ for some constant $\gamma<1$. This implies $\alpha_{k}=\gamma \cdot\left(\lambda_{1}^{k}-\nu_{1}^{k}\right)$.

The vertices of the triangle $f_{1}^{k}(T)$ are $e_{1}$ and $f_{1}^{k}(0)=\left(1-\lambda_{1}^{k}\right) e_{1}$ and

$$
f_{1}^{k}\left(e_{2}\right)=\left(\begin{array}{c}
\alpha_{k}+1-\lambda_{1}^{k} \\
\nu_{1}^{k}
\end{array}\right)=\left(\begin{array}{l}
y_{1} \\
y_{2}
\end{array}\right) \text {. }
$$


For the angle $\beta_{k}$ at $e_{1}$ we have $\tan \beta_{k}=y_{2} /\left(1-y_{1}\right)=\nu_{1}^{k} /\left(\lambda_{1}^{k}-\alpha_{k}\right)$. If $\lambda_{1}=\nu_{1}$ then $\tan \beta_{k}=\lambda_{1} /\left(\lambda_{1}-\alpha k\right)$ with $\alpha<0$. For the case $\lambda_{1}>\nu_{1}$ we get $\tan \beta_{k}=$ $\nu_{1}^{k} /\left(\lambda_{1}^{k}(1-\gamma)+\nu_{1}^{k} \gamma\right)$. In both cases, $\beta_{k}$ converges to 0 .

For $e_{2}$, the proof is similar. Now consider $z=f_{1}\left(e_{2}\right)=f_{2}\left(e_{1}\right)$. Since affine maps preserve tangents of curves, $f_{1}\left(\left[0, e_{2}\right]\right)=\left[z,\left(1-\lambda_{2}\right) e_{2}\right]$ is a right-sided tangent of $J$ at $z$, and $f_{2}\left(\left[0, e_{1}\right]\right)=\left[z,\left(1-\lambda_{1}\right) e_{1}\right]$ is a left-sided tangent at $z$. By the same argument, all points $f_{\mathbf{j}}(z)$ with $\mathbf{j} \in I^{*}$ admit one-sided tangents. These are the points where two pieces of $J$ meet.

Proof of Theorem 2. (i) We show that if $\alpha=\lambda_{1}+\nu_{2}-1<0$ and $\beta=\lambda_{2}+\nu_{1}-1<0$, then $J$ admits tangents at all points which are not endpoints of little pieces. This is done with Proposition 12 below.

(ii) Since $z=\left(\begin{array}{c}\nu_{2} \\ \nu_{1}\end{array}\right)$, Proposition 11 says that the one-sided tangents at $z$ have slope $\left(1-\lambda_{2}-\nu_{1}\right) / \nu_{2}$ and $\nu_{1} /\left(1-\lambda_{1}-\nu_{2}\right)$, respectively. If $\nu_{1} \nu_{2}=\left(1-\lambda_{1}-\nu_{2}\right)\left(1-\lambda_{2}-\nu_{1}\right)$ then the one-sided tangents agree, and $J$ admits tangents at $z$ and all $f_{\mathbf{j}}(z)$. Thus $J$ is a differentiable curve. The continuity of the derivative is shown in Remark 15 below.

Proposition 12. If $\alpha<0$ and $\beta<0$ then the curve $J$ has tangents at all points which are not endpoints of pieces.

Proof. For Proposition 9, we proved that all chords $y-y^{\prime}$ with $y, y^{\prime} \in J$ lie in the cone $W=\left\{\left(\begin{array}{c}w_{1} \\ w_{2}\end{array}\right) \mid w_{1} w_{2} \leq 0\right\}$ given by the second and fourth quadrant. For the matrices $M_{i}$ of $f_{i}$ we have $M_{i} W \subseteq W, i=1,2$. Thus the chords between points of $J_{i_{1} \ldots i_{n}}$ lie in $M_{i_{1}} \ldots M_{i_{n}} W \subseteq W$. Now we show that for each sequence $\mathbf{i}=i_{1} i_{2} \ldots \in I^{\infty}$ the cones $M_{i_{1}} \ldots M_{i_{n}} W$ converge to a single line $L=L(\mathbf{i})$ for $n \rightarrow \infty$. Then $L+x$ is the tangent of $J$ at $x=\pi(\mathbf{i})$.

For sequences $\mathbf{i}$ which end with $\overline{1}=111 \ldots$ or with $\overline{2}$ this can be shown as in Proposition 11 (see Remark 14). But we obtain only one-sided tangents since $x$ was an endpoint of $J_{i_{1} \ldots i_{n}}$ for all sufficiently large $n$. Now we consider an arbitrary point $x=\pi(\mathbf{i})$ which is not an endpoint of any small piece of $J$, and prove there is a two-sided derivative. Since $\mathbf{i}$ does not end with $\overline{1}$ or $\overline{2}$, there exist positive integers $1<k_{1}<k_{2}<\ldots$ such that $i_{k_{m}}=1$ and $i_{k_{m}+1}=2$ for $m=1,2, \ldots$

The size of a subcone $W^{\prime} \subseteq W$ is taken as diam $\left(\left[-e_{1}, e_{2}\right] \cap W^{\prime}\right)$. It will be helpful to define the linear functional $\phi(w)=w_{2}-w_{1}$, and take the line $\ell=\{w \mid \phi(w)=1\}$ instead of $\left[-e_{1}, e_{2}\right]$. We shall find a constant $\delta>0$ such that

$$
\operatorname{diam}\left(\ell \cap M_{i_{k_{1}}} \ldots M_{i_{k_{m}-1}} W\right) \leq(1-\delta)^{m-1} \operatorname{diam}(\ell \cap W)
$$

for all $m$. Then $M_{i_{k_{1}}} \ldots M_{i_{k_{m}-1}} W$ converges to a line for $m \rightarrow \infty$, and the proof is complete.

Let $V=M_{1} M_{2} W$. Since $\alpha, \beta<0$ and

$$
M_{1} M_{2}=\left(\begin{array}{cc}
\lambda_{1} & \alpha \\
0 & \nu_{1}
\end{array}\right)\left(\begin{array}{cc}
\nu_{2} & 0 \\
\beta & \lambda_{2}
\end{array}\right)=\left(\begin{array}{cc}
\lambda_{1} \nu_{2}+\alpha \beta & \alpha \lambda_{2} \\
\nu_{1} \beta & \nu_{1} \lambda_{2}
\end{array}\right) .
$$


both $M_{1} M_{2}\left(-e_{1}\right)$ and $M_{1} M_{2} e_{2}$ are in int $W$ and hence $V \subset \operatorname{int} W$. Consequently,

$$
\delta:=\frac{\operatorname{dist}(\ell \cap V, \ell \cap \partial W)}{\operatorname{diam}(\ell \cap W)}=\frac{\operatorname{dist}\left(\left[-e_{1}, e_{2}\right] \cap V,\left\{-e_{1}, e_{2}\right\}\right)}{\operatorname{diam}\left[-e_{1}, e_{2}\right]}>0,
$$

and $\operatorname{diam}(\ell \cap V) \leq(1-2 \delta) \cdot \operatorname{diam}(\ell \cap W)$.

Lemma 13. If $B$ is a regular $2 \times 2$ matrix with $B W \subseteq W$ then

$$
\operatorname{diam}(\ell \cap B V) \leq(1-\delta) \cdot \operatorname{diam}(\ell \cap B W) .
$$

Proof of Lemma. Let $\ell^{\prime}=B(\ell)$. Since linear maps preserve length ratios within lines, we have

$$
\delta=\frac{\operatorname{dist}\left(\ell^{\prime} \cap B V, \ell^{\prime} \cap B(\partial W)\right)}{\operatorname{diam}\left(\ell^{\prime} \cap B W\right)}
$$

$\ell^{\prime} \cap B(\partial W)$ consists of two points $-B e_{1}, B e_{2}$ which we call $a$ and $b$, choosing the order so that $\phi(a) / \phi(b) \geq 1$. Furthermore, let $c$ be the point in $\ell^{\prime} \cap B V$ which assumes maximum distance from $a$. Then $c=\tau a+(1-\tau) b$ with $\tau \in[\delta, 1-\delta]$ because of (5).

The projection $p(w)=w / \phi(w)$ maps $[a, b]$ onto $\ell \cap B W$, and $\ell \cap B V$ is contained in $[p(a), p(c)]$. Thus

$$
\begin{aligned}
\operatorname{diam}(\ell \cap B V) & \leq|p(c)-p(a)|=\left|\frac{\tau a+(1-\tau) b}{\tau \phi(a)+(1-\tau) \phi(b)}-\frac{a}{\phi(a)}\right| \\
& =\left|\frac{\tau a+(1-\tau) b-\tau a-(1-\tau) a \cdot \phi(b) / \phi(a)}{\tau \phi(a)+(1-\tau) \phi(b)}\right| \\
& =\frac{(1-\tau) \cdot|b / \phi(b)-a / \phi(a)|}{\tau \phi(a) / \phi(b)+(1-\tau)} \\
& \leq(1-\delta) \cdot|p(a)-p(b)|=(1-\delta) \cdot \operatorname{diam}(\ell \cap B W) .
\end{aligned}
$$

To finish the proof of Proposition 12, we show (4) by induction on $m$. Let $B_{m}=\prod_{k=1}^{k_{m}-1} M_{i_{k}}$. The matrix $C_{m}=\prod_{k=k_{m}}^{k_{m+1}-1} M_{i_{k}}$ has the form $C_{m}=M_{1} M_{2} \cdots$, so $C_{m} W \subset M_{1} M_{2} W=V$ and $B_{m+1} W=B_{m} C_{m} W \subset B_{m} V$ for $m=1,2, \ldots$ We apply the Lemma to $B=B_{m}$ and obtain

$$
\operatorname{diam}\left(\ell \cap B_{m+1} W\right) \leq \operatorname{diam}\left(\ell \cap B_{m} V\right) \leq(1-\delta) \cdot \operatorname{diam}\left(\ell \cap B_{m} W\right)
$$

for $m \geq 1$. This implies (4).

Remark 14. Assuming $\alpha, \beta<0$, we proved that for each address sequence $i_{1} i_{2} \ldots$ which does not end with $\overline{1}$ or $\overline{2}$, and the corresponding point $x=\pi\left(i_{1} i_{2} \ldots\right)$ there is the tangent line $L(x)=\bigcap_{n=1}^{\infty} M_{i_{1} \ldots i_{n}} W$. This is also true for $i_{1} i_{2} \ldots=\overline{1}$ or $\overline{2}$, hence for all addresses.

Proof. The cones $M_{1}^{n}(W)$ form a decreasing sequence, and their intersection is a closed convex cone in $\mathbb{R}^{2}$. If this is not the line $r e_{1}, r \in \mathbb{R}$, the intersection cone has a second bounding line determining another eigenvector of $M_{1}$. However, the second eigenvector $\left(\begin{array}{c}-\alpha \\ \lambda-\nu\end{array}\right)$ of $M_{1}$ does not belong to $W$. Note that $\alpha<0$ is needed 
here. In Proposition 11 we used a subcone of $W$. The proof for $\overline{2}$ uses $\beta<0$, and $M_{i_{1} \ldots i_{n} \overline{1}}=M_{i_{1} \ldots i_{n}} L\left(e_{1}\right)$ is a line.

Remark 15. For each address $i_{1} i_{2} \ldots$ of $x \in J$ the $M_{i_{1} \ldots i_{n}} W$ shrink down to a line $L(x)$. Only when $i_{1} i_{2} \ldots$ ends with $\overline{1}$ or $\overline{2}$, there is a second address of $x$ and a second line $L^{\prime}(x)$. So for each $\epsilon>0$ there is $n$ such that diam $\left(\ell \cap M_{i_{1} \ldots i_{n}} W\right)<\epsilon$. Thus all tangents $L(y)$ (and also $L^{\prime}(y)$ ) with $y \in J_{i_{1} \ldots i_{n}}$ are within distance $\epsilon$ from $L(x)$. If $x$ is not an endpoint of a piece of $J$, the $J_{i_{1} \ldots i_{n}}$ are neighborhoods of $x$, and the derivative is continuous at $x$.

For endpoints, we have one-sided neighborhoods, and one-sided continuity. If now the one-sided tangents at $z$ coincide, then $L(x)=L^{\prime}(x)$ for all points with two addresses, we can put the one-sided neighborhoods together, and the derivative becomes a continuous function.

\section{The UniQUeness of THE PARABOla}

One can ask whether our $C^{1}$ self-affine functions are even $C^{2}$. There is at least one well-known example, the parabola. Parabolic arcs play the same role for self-affine sets as intervals do for self-similar sets. The parabola $x_{2}=x_{1}^{2} / 2$ admits a transitive group of 'parabolic translations' $\phi\left(\left(\begin{array}{l}x_{1} \\ x_{2}\end{array}\right)\right)=\left(\begin{array}{c}x_{1}+t \\ t x_{1}+x_{2}+t^{2} / 2\end{array}\right), t \in \mathbb{R}$, as well as 'parabolic homotheties' $\psi\left(\left(\begin{array}{l}x_{1} \\ x_{2}\end{array}\right)\right)=\left(\begin{array}{c}\lambda x_{1} \\ \lambda^{2} x_{2}\end{array}\right)$ with $\lambda>0$. Thus any arc $P$ of a parabola can be mapped by an affine map into any other one, and for every division $P=P_{1} \cup P_{2}$ into two subarcs, generated by an interior point $z$ of $P$, we have a representation of $P$ as a self-affine set.

Example 16. In our coordinate system, the symmetric representation of a parabolic arc $P$ is given by $\lambda_{i}=\frac{1}{2}, \nu_{i}=\frac{1}{4}$. The following one-parameter family provides all representations of $P$ (cf. Figure 2 for $\lambda_{1}=0.4$ ).

$$
\lambda_{1}+\lambda_{2}=1, \quad \nu_{1}=\lambda_{1}^{2}, \quad \nu_{2}=\lambda_{2}^{2}
$$

Proof. A parabola is defined by two of their points together with their tangents, so $P$ is uniquely determined. In the coordinate system of Figure 1 it has equation $x_{2}=\frac{1}{2}\left(x_{1}^{2}+1\right)$. This is $2 w_{1}+2 w_{2}-1=\left(w_{1}-w_{2}\right)^{2}$ in our coordinate system since $x_{1}=w_{1}-w_{2}, x_{2}=w_{1}+w_{2}$. Solving the quadratic equation for $w_{2}$ we get

$$
w_{2}=w_{1}+1-2 \sqrt{w_{1}}=\left(1-\sqrt{w_{1}}\right)^{2} \quad, \quad \text { or } \quad \sqrt{w_{1}}+\sqrt{w_{2}}=1 .
$$

The derivative is $w_{2}^{\prime}=1-1 / \sqrt{w_{1}}$. Now consider the tangent line to $P$ at an arbitrary point $\left(\begin{array}{c}\nu_{2} \\ \nu_{1}\end{array}\right)$. It has equation $w_{2}=\nu_{1}+\left(w_{1}-\nu_{2}\right)\left(1-1 / \sqrt{\nu_{2}}\right)$. This line passes through $w_{1}=0, w_{2}=1-\lambda_{2}$ which yields

$$
1-\lambda_{2}=\nu_{1}-\nu_{2}+\sqrt{\nu_{2}}=1-\sqrt{\nu_{2}} \quad \text { because of } \quad \nu_{1}=1+\nu_{2}-2 \sqrt{\nu_{2}} .
$$

Thus $\lambda_{2}=\sqrt{\nu_{2}}$, and similarly $\lambda_{1}=\sqrt{\nu_{1}}$, since the tangent line also passes through $w_{1}=1-\lambda_{1}, w_{2}=0$. And $\sqrt{\nu_{1}}+\sqrt{\nu_{2}}=1$ was already proved.

Proof of Theorem 3, (i). We have a plane $C^{2}$ curve $J$ parametrized by $x=\phi(t)$ with $\phi(0)=0$ and $\phi^{\prime \prime}(0) \neq 0$, and a contractive linear map $h \neq 0$ which maps $J$ into itself. We show that $J$ is contained in a parabola. The condition $\phi^{\prime \prime}(0) \neq 0$ does 
not depend on $\phi$. It says that the curvature of $J$ at 0 is nonzero. In particular, any subarc of $J$ with the point 0 cannot be contained in a line.

Since $h(J) \subseteq J$ and $h(0)=0$, the map $h$ fixes the tangent line of $J$ at 0 . This must be an eigenvector of $h$. We use the Jordan normal form of $h$. The classes of $C^{1}$ and $C^{2}$ curves, as well as the class of parabolas, and a curve's property of having nonzero curvature at a point, are preserved under linear transformations. For that reason, we can calculate with the Jordan base as standard coordinate system. Moreover, we assume that the first basis vector is an eigenvector of $h$ in the direction of the tangent of $J$ at 0 . Thus $h$ has a matrix of the form $\left(\begin{array}{ll}\lambda & \gamma \\ 0 & \nu\end{array}\right)$ where $\gamma=0$ if $\lambda \neq \nu$. Note that $|\lambda|,|\nu|<1$ since $h$ is contractive.

In the following, we use coordinates $x, y$ instead of $x_{1}, x_{2}$. Let us take a $C^{2}$ parametrization $\varphi(t)=\left(\begin{array}{l}x(t) \\ y(t)\end{array}\right)$ of $J$ in our new coordinate system with $\varphi(0)=0$ and $\varphi^{\prime}(0) \neq 0$ (the parametrization by arc length fulfils this condition). Then $x^{\prime}(0) \neq 0$ since $y^{\prime}(0)=0$ by the choice of the first axis. So there exists a $\delta>0$ such that $x^{\prime}(t) \neq 0$ for $0 \leq t<\delta$. Thus $x(t)$ is a monotone $C^{2}$ function for $0 \leq t<\delta$.

By the inverse function theorem, the inverse function $t(x)$ is $C^{2}$ for $0 \leq x<\epsilon=$ $x(\delta)$, and the composition $y(t(x))$ is also $C^{2}$ for $0 \leq x<\epsilon$. This function which describes $J$ in the vicinity of 0 will now be called $y=y(x)$, and $t$ is eliminated. We have $y(0)=0, y^{\prime}(0)=0$, and $y^{\prime \prime}(0) \neq 0$, since otherwise the curvature of $J$ at 0 would be zero. Now we apply the assumption $h(J) \subseteq J$. From

$$
\left(\begin{array}{ll}
\lambda & \gamma \\
0 & \nu
\end{array}\right) \cdot\left(\begin{array}{c}
x \\
y(x)
\end{array}\right)=\left(\begin{array}{c}
\lambda x+\gamma y(x) \\
\nu y(x)
\end{array}\right)=\left(\begin{array}{c}
z \\
y(z)
\end{array}\right)
$$

with $z=z(x)=\lambda x+\gamma y(x)$ we get the derivatives

$$
\begin{gathered}
\nu y^{\prime}(x)=y^{\prime}(z) \cdot z^{\prime}(x) \quad \text { with } z^{\prime}(x)=\lambda+\gamma y^{\prime}(x) \quad \text { and } \\
\nu y^{\prime \prime}(x)=y^{\prime \prime}(z) \cdot z^{\prime}(x)^{2}+y^{\prime}(x) \cdot z^{\prime \prime}(x) \quad \text { with } z^{\prime \prime}(x)=\gamma y^{\prime \prime}(x)
\end{gathered}
$$

for $0 \leq x<\epsilon$. Inserting $x=0$ and $z=0, y^{\prime}=0, z^{\prime}=\lambda$ in (6) we obtain $\nu=\lambda^{2}$ since $y^{\prime \prime}(0) \neq 0$. Thus $\gamma=0$ since $\nu$ and $\lambda$ are different. Equation (6) now simplifies:

$$
y^{\prime \prime}(x)=y^{\prime \prime}(\lambda x) \quad \text { and hence } y^{\prime \prime}(x)=y^{\prime \prime}\left(\lambda^{n} x\right)
$$

for $x \in[0, \epsilon)$ and $n=1,2, \ldots$ For the limit $n \rightarrow \infty$ we obtain $y^{\prime \prime}(x)=y^{\prime \prime}(0)$ since $|\lambda|<1$ and $y^{\prime \prime}$ is continuous. Thus $y(x)$ is a quadratic function for $0 \leq x<\epsilon$. Applying $h^{-1}$ several times to that part of $J$, we see that the arc $J$ is contained in a parabola.

Proof of Theorem 3, (ii). If $J=f_{1}(J) \cup \ldots \cup f_{m}(J)$ is a self-affine curve and $J_{\mathbf{i}}$ with $\mathbf{i}=i_{1} \ldots i_{n}$ is a small piece of $J$, then $f_{\mathbf{i}}$ is a contracting affine map with fixed point in $J_{\mathbf{i}}$ which maps $J$ into itself.

Now assume $J \subset R^{2}$ is a $C^{2}$ curve and not subset of a line, so that $\phi^{\prime \prime}(x) \neq 0$ for some parametrization of $J$ and some $x \in J$. Since $\phi^{\prime \prime}$ is continuous, this implies $\phi^{\prime \prime}(y) \neq 0$ for all $y$ in a small piece $J_{\mathbf{i}}$ containing $x$. Shifting our coordinate system to the fixed point of $f_{\mathbf{i}}=h$ and applying part (i), we see that $J$ is contained in a segment or parabola. 


\section{REFERENCES}

[1] V. V. Aseev, A. V. Tetenov and A. S. Kravchenko, On Selfsimilar Jordan Curves on the Plane, Siberian Math. J. 44, No. 3 (2003), 379-386.

[2] C. Bandt and H. Rao, Topology and separation of self-similar fractals in the plane, Nonlinearity 20 (2007), 1463-1474.

[3] M.F.Barnsley, Fractals Everywhere, 2nd ed., Academic Press 1993

[4] K.J. Falconer, The geometry of fractal sets, Cambridge University Press, 1985.

[5] K.J. Falconer, Fractal Geometry, Wiley 1990

[6] J.E. Hutchinson, Fractals and self-similarity, Indiana Univ. Math. J. 30 (1981), 713-747.

[7] A. Käenmäki, On the geometric structure of the limit set of conformal iterated function systems. Publ. Mat. 47 (1) (2003), 133-141.

[8] A. Käenmäki, Geometric rigidity of a class of fractal sets, Math. Nachr. 279 (2006), 179-187.

[9] A. Käenmäki and M. Vilppolainen, Dimension and measures on sub-self-affine sets, Monatshefte Math. 2010

[10] A. Kravchenko, Smooth self-affine curves (in Russian), Preprint No. 161, Sobolev Institute of Mathematics, Siberian Branch of the Russian Academy of Sciences, 2005.

[11] P. Mattila, Geometry of Sets and Measures in Euclidean Spaces, Cambridge University Press 1995.

[12] P.A.P. Moran, Additive functions of intervals and Hausdorff measure. Proc. Cambridge Philos. Soc. 42, (1946) 15-23. 